\title{
Quantum simulation of Cayley-tree Ising Hamiltonians with three-dimensional Rydberg atoms
}

\author{
Yunheung Song, ${ }^{*}$ Minhyuk Kim, Hansub Hwang, Woojun Lee, ${ }^{\dagger}$ and Jaewook Ahn $\oplus^{\ddagger}$ \\ Department of Physics, Korea Advanced Institution of Science and Technology, Daejeon 34141, Republic of Korea
}

(Received 17 November 2020; accepted 17 March 2021; published 29 March 2021)

\begin{abstract}
Significant efforts are being directed toward developing a quantum simulator capable of solving combinatorial optimization problems. The challenges are Hamiltonian programming in terms of high-dimensional qubit connectivities and large-scale implementations. Here, we report a quantum simulation demonstration of Ising Hamiltonians with up to $N=22$ spins mapped on various Cayley-tree graphs. For this, we use three-dimensional arrangements of Rydberg single atoms arranged in such a way that their Rydberg atoms and blockaded strong couplings respectively represent the vertices and edges of each graph. Three different Cayley-tree graphs of $Z=3$ neighbors and of up to $S=4$ shells are constructed, and their many-body ground states and Néel's order formations are experimentally probed. The antiferromagnetic phase in regular Cayley trees and frustrated competing ground states in a dual-center Cayley tree are directly observed, demonstrating the possibilities of high-dimensional qubit connections in quantum simulators.
\end{abstract}

DOI: 10.1103/PhysRevResearch.3.013286

\section{INTRODUCTION}

In recent years, quantum simulations have received significant attention because quantum annealing in particular has the potential to solve complex computational problems which are often intractable with nonquantum computational methods [1-4]. Quantum annealing is a procedure of making Hamiltonian $H(t)$ of a quantum many-body system adiabatically evolve from $\hat{H}_{i}$ to $\hat{H}_{f}$,

$$
\hat{H}(t)=\hat{H}_{i}-\frac{t}{t_{f}}\left(\hat{H}_{i}-\hat{H}_{f}\right),
$$

so that the quantum state $|\Psi(t)\rangle$ initially prepared in the ground state of the former reaches the ground state of the latter. Quantum annealing machines are considered with superconducting qubits [5-8] and trapped-ion qubits [9-12], aiming for various combinatorial optimization problems such as quantum simulations [13], classfications [14], and planning [15]. While many efforts in quantum annealing are being focused on large-scale implementations [16-20] toward quantum speedup [21-24], here, we explore the possibility of high-dimensional qubit connectivities. Theoretical proposals emphasize and thus utilize qubit connectivities for

\footnotetext{
* Present address: Department of Physics, University of WisconsinMadison, 1150 University Avenue, Madison, Wisconsin 53706, USA.

${ }^{\dagger}$ Present address: Department of Computer Science and Engineering, Seoul National University, Seoul 08826, Republic of Korea.

¥jwahn@kaist.ac.kr
}

Published by the American Physical Society under the terms of the Creative Commons Attribution 4.0 International license. Further distribution of this work must maintain attribution to the author(s) and the published article's title, journal citation, and DOI. nondeterministic polynomial time (NP)-hard optimization problems: for example, the Lechner-Hauke-Zoller scheme $[25,26]$ proposed a quantum annealing architecture for allto-all connectivities using local interactions, and quantum optimization protocols are considered, e.g., for maximum independent set problems utilizing the nature of long-range couplings especially in Rydberg-atom quantum simulators [27]. In the context relevant to this paper, Rydberg-atom quantum simulators [28,29] draw attention because of their high tunability in qubit connectivities [30-34] as well as manybody controllability in adiabatic processes [35-39].

In this paper, we consider, as a prototypical fractal structure, Cayley-tree graphs of neutral atom arrangements in which atoms and strongly interacting atom pairs respectively represent vertices and edges of the graphs (see Fig. 1). Cayley trees are homogeneous and isotropic tree graphs of a fixed number of edges and no loop [40,41]. Their infinite version is a Bethe lattice, widely used in various physics areas as a fundamental theoretical platform, often providing exactly solvable models in classical and quantum problems [42]. In experiments to be described below, we use three-dimensional (3D) arrangements of neutral atoms to construct atomic Cayley graphs of coordination number $Z=3$ and shell number up to $S=4$, as in Fig. 1(a), and run quantum simulation to probe the many-body ground states of the corresponding Cayley-tree Ising-Hamiltonians.

\section{CAYLEY-TREE ATOM ARRANGEMENTS IN 3D SPACE}

Neutral atoms (rubidium, ${ }^{87} \mathrm{Rb}$ ) are arranged in 3D space with optical tweezers (far-off resonant optical dipole traps) $[34,43]$ (see Sec. VI for technical details). Three different Cayley-tree graphs are constructed. The first one is the threeshell $(S=3)$ Cayley tree, shown in Fig. 1(b), which can be denoted by $G_{10}=(0 s)(1 s)^{3}(2 s)^{6}$, having one atom in the first (center) shell, three in the second shell, and six in the third 
(a)

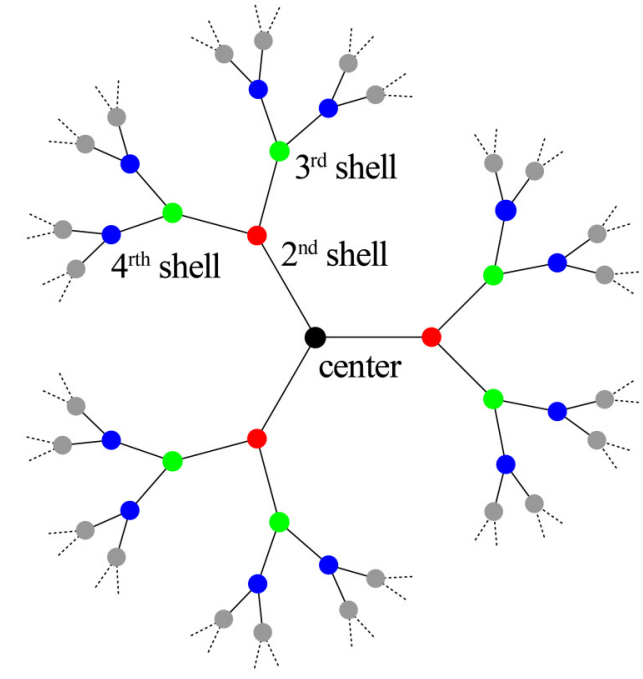

(b)

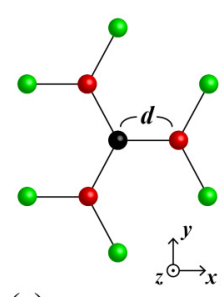

(e)

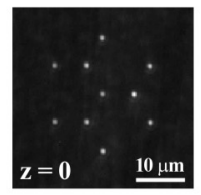

(c)

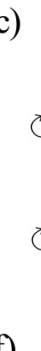

(f)

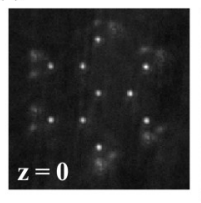

(d)

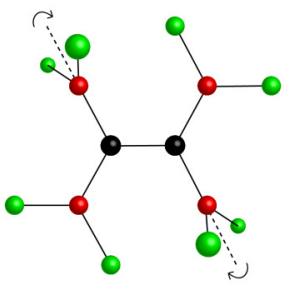

(g)

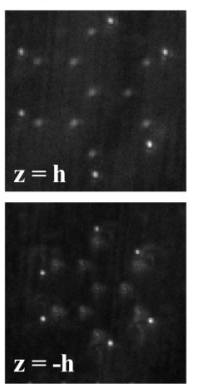

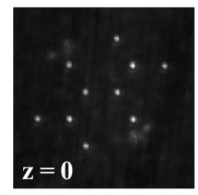
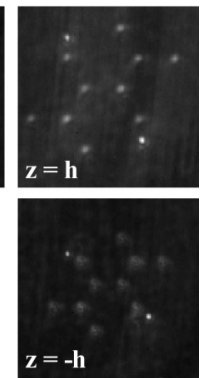

FIG. 1. (a) A generic Cayley-tree graph of coordination number $Z=3$, with vertices and edges representing atoms and Rydberg-blockaded atom pairs, respectively. (b) A three-shell Cayley tree $\left(G_{10}\right)$ of 10 atoms of interatom distance $d$. (c) A four-shell Cayley tree $\left(G_{22}\right)$ constructed in three planes at $z=0, \pm h(h=d / 1.2)$, where the last-shell branches are rotated by $72^{\circ}$ to avoid unwanted couplings. (d) A dual-center Cayley tree $\left(G_{14}\right)$ of 14 atoms. (e)- $(\mathrm{g})$ Plane-by-plane fluorescence images of the corresponding atom arrangements.

shell. The second one is the four-shell $(S=4)$ Cayley tree, $G_{22}=(0 s)(1 s)^{3}(2 s)^{6}(3 s)^{12}$, having 10 atoms in $G_{10}$ and 12 in the fourth shell, as in Fig. 1(c). The last one is $G_{14}=$ $(0 s)^{2}(1 s)^{4}(2 s)^{8}$, a dual-center Cayley tree, having two firstshell atoms, four second-shell atoms, and eight third-shell atoms, as in Fig. 1(d).

In our atom arrangements, each atom represents a vertex of a Cayley-tree graph and each pair of strongly-interacting atoms an edge. Nonconnected atoms are supposed to interact with each other much weaklier than connected atoms. The Hamiltonian of these atoms being coherently and simultaneously excited to a Rydberg energy state is given (in units of $\hbar=1)$ by

$$
\hat{H}=\frac{1}{2} \sum_{j=1}^{N}\left[\Omega \hat{\sigma}_{x}^{(j)}-\Delta \hat{\sigma}_{z}^{(j)}\right]+\sum_{j<k} U_{j k} \hat{n}^{(j)} \hat{n}^{(k)},
$$

where $N$ is the number of atoms, $\Omega$ is the Rabi frequency, $\Delta$ is the detuning, and $U_{j k}=C_{6} /\left|\vec{r}_{j}-\vec{r}_{k}\right|^{6}$ is the pairwise atom interaction in the van der Waals interaction regime $[37,38]$. Pauli operators $\hat{\sigma}_{x, z}$ are defined for a pseudospin $\frac{1}{2}$ system composed of the ground state $|\downarrow\rangle=\left|5 S_{1 / 2}, F=2, m_{F}=2\right\rangle$ and Rydberg state $|\uparrow\rangle=\left|71 S_{1 / 2}, m_{J}=\frac{1}{2}\right\rangle$ of each atom, and $\hat{n}=(1+$ $\left.\hat{\sigma}_{z}\right) / 2$. For the strong and weak interactions of connected and nonconnected atom pairs, respectively, we set the distances of connected atoms the same and within the Rydberg-blockade radius, i.e., $\left|\vec{r}_{j}-\vec{r}_{k}\right|=d<r_{b} \equiv\left(C_{6} / \hbar \Omega\right)^{1 / 6}=9.8 \mu \mathrm{m}$, for $(j, k) \in E$ (the edge set of a graph $G$ ), and the distances of all others are $\left|\vec{r}_{j}-\vec{r}_{k}\right|>r_{b}$.

The two-dimensional (2D) arrangement of $G_{10}$ is made with $d<r_{b}<\sqrt{3} d$ to satisfy the above condition, in which, as shown in Fig. 1(e), the last-shell atoms of different branches are separated more than $\sqrt{3} d$, having significantly weaker interactions (at most $\frac{1}{27}$ times smaller) than $U \equiv C_{6} / d^{6}$ of the connected atom pairs. However, $G_{22}$ and $G_{14}$ cannot be constructed to be planar because, in $2 \mathrm{D}$ arrangements, atoms of different outermost branches are too close, requiring nonplanar, 3D arrangements. As shown in Fig. 1(c), we rotate the last-shell branches of $G_{22}$ by an angle of $2 \pi / 5$ about the axes along the previous branches, so that all the last-shell atoms are well separated (more than $\sqrt{3} d$ ) from each other. In $G_{14}$, some last-shell branches are rotated similarly, as shown in Fig. 1(d). As-constructed atom arrangements are shown in Figs. $1(\mathrm{e})-1(\mathrm{~g})$, respectively, for $G_{10}, G_{22}$, and $G_{14}$.

\section{PHASE DIAGRAMS OF CAYLEY-TREE ISING SPINS}

With atoms arranged on one of the above Cayley-tree graphs, we perform quantum simulation to find the manybody ground state of a target Hamiltonian. The atoms are initially prepared in $|\downarrow \downarrow \cdots \downarrow\rangle$ (the para-magnetic down spins, which we denote by Phase I), which is the ground state of the initial Hamiltonian $\hat{H}(\Omega=0, \Delta<0,|U| \ll|\Delta|)$ in Eq. (2). The target (final) Hamiltonian is

$$
\hat{H}_{G}\left(U, \Delta_{f}\right)=U \sum_{(j, k) \in E} \hat{n}^{(j)} \hat{n}^{(k)}-\frac{\Delta_{f}}{2} \sum_{j=1}^{N} \hat{\sigma}_{z}^{(j)},
$$

where $E$ is the edge set of $G \in\left\{G_{10}, G_{22}, G_{14}\right\}, \Delta_{f}$ is the final detuning, and no couplings are assumed for unedged atom pairs. We note that $\hat{H}_{G}$ is an Ising spin-glass Hamiltonian, given by

$$
\hat{H}_{G}=J \sum_{(j, k) \in E} \hat{\sigma}_{z}^{(j)} \hat{\sigma}_{z}^{(k)}+h_{z}^{C} \sum_{j \in C} \hat{\sigma}_{z}^{(j)}+h_{z}^{V} \sum_{j \in V} \hat{\sigma}_{z}^{(j)}
$$

where $J=U / 4$ is the coupling, $h_{z}^{C}=3 U / 4-\Delta_{f} / 2$ and $h_{z}^{V}=$ $U / 4-\Delta_{f} / 2$ are the local fields, and $C$ and $V$ denote the vertices in the core (inner shells) and valence (outermost) shell, respectively, of a Cayley-tree graph $G(E, C+V)$. In general, as the ground states of an Ising Hamiltonioan depend on the 
(a)

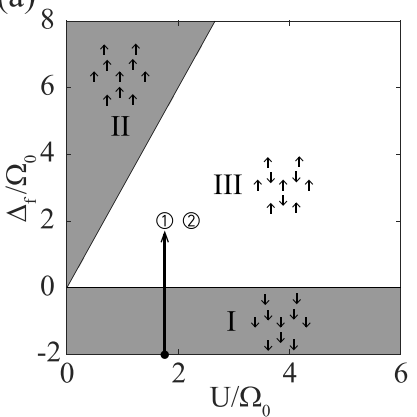

(b)

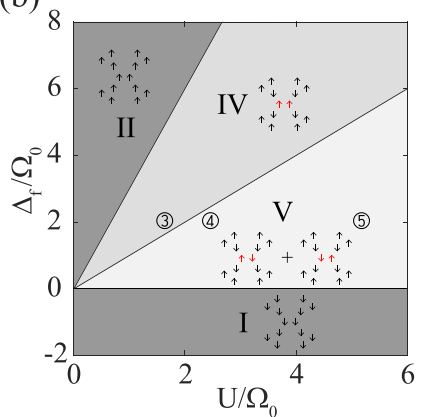

FIG. 2. Phase diagrams of Cayley-tree Ising Hamiltonians (a) $\hat{H}_{G_{10}}$ and $\hat{H}_{G_{22}}$ and (b) $\hat{H}_{G_{14}}$, in which ground-state spin configurations are paramagnetic down spins (Phase I), para-magnetic up spins (Phase II), antiferromagnetic phase (Phase III), and antiferrolike phases with center spins $|\uparrow \uparrow\rangle$ (Phase IV) and $(|\uparrow \downarrow+\downarrow \uparrow\rangle) / \sqrt{2}$ (Phase V). Circled numbers indicate the Hamiltonian parameters of the experimental data in Figs. 3 and 4: (1) $\left(U / \Omega_{0}, \Delta_{f} / \Omega_{0}\right)=(1.82$, 2) in Phase III of $G_{10}$, (2) $(2.25,2)$ in Phase III of $G_{22}$, (3) $(1.67,2)$ in Phase IV of $G_{14}$, (4) $(2.70,2)$, and (5) $(5.41,2)$ in Phase V of $G_{14}$. $\Omega_{0}=1.1(2 \pi) \mathrm{MHz}$ in all experiments.

specific atom arrangement of $G$, finding such arrangementspecific ground states of an arbitrary Ising-spin graph is an NP-complete problem [44].

However, Cayley-tree graphs allow heuristic understanding of their phase diagrams in Fig. 2 as follows: Cayley trees have more atoms on the valence shell than on the rest (the inner shells). Therefore, with Hamiltonian $\hat{H}_{G}$ in Eq. (3) (of positive coupling $U>0$ in our consideration), the valence spins are all aligned either up or down depending upon $\Delta_{f}>0$ or $\Delta_{f}<0$, respectively, resulting in the $\Delta_{f}=0$ phase boundary. For $\Delta_{f}<0$, inner-shell spins, adjacent to the valence spins, also favor down spins, as their couplings $U \hat{n}^{(j)} \hat{n}^{(k)}$ to adjacent outer-shell down spins are zero, which subsequently results in all down spins (so paramagnetic down spins, Phase I). For $\Delta_{f}>0$, single-spin flipping energy $\left(\Delta_{f}\right)$ competes with adjacent antiferromagnetic couplings. For $\Delta_{f}>3 U$, the former is always higher than the latter (of $\max 3 U$ ), so all spins are up (paramagnetic up spins, Phase II). In between, $0<\Delta_{f}<3 U$, the antiferromagnetic coupling is stronger, thus favoring the antiferromagnetic phase (Phase III) of shellby-shell alternating spins. The resulting $\hat{H}_{G}$ phase diagram is shown for $G_{10}$ in Fig. 2(a), in which the antiferromagnetic phase (Phase III) has up spins on the first and third shells and down spins on the second. The phase diagram for $G_{22}$ differs from $G_{10}$ only in Phase III, where the odd-numbered shells are down spins and the even-numbered shells are up spins. In short, the phase diagram of $\hat{H}_{G}$ (of positive $U$ ) for regular Cayley graphs ( $G_{10}$ and $G_{22}$ in our consideration) has the para-antiferro phase boundaries determined by $\Delta_{f}=3 U$ and $\Delta_{f}=0$, so the antiferromagnetic ordering is expected in the region $0<\Delta_{f}<3 U$ (Phase III), as in Fig. 2(a).

The phase diagram for $G_{14}$, the dual-center Cayley tree, is a little more complex than regular Cayley trees because of the frustrations of the center spins. Exact diagonalization of $\hat{H}_{G_{14}}$ finds the phase diagram as shown in Fig. 2(b), in which the paramagnetic phases (Phases I and II) are the same as $G_{10}$, but Phase III is split to Phases IV and V of respective

center-spin configurations of $|\uparrow \uparrow\rangle$ and $(|\uparrow \downarrow+\downarrow \uparrow\rangle) / \sqrt{2}$. The new phase boundary is $U=\Delta_{f}$, along which the single-spin flipping cost equals the additional frustration cost of the center spins. It is noted that, in Phase V, other energy-degenerate ground states, with respective center-spin configurations of $|\uparrow \downarrow\rangle,|\downarrow \uparrow\rangle$, and $(|\uparrow \downarrow-\downarrow \uparrow\rangle) / \sqrt{2}$, are all dark states (not coupled by $\hat{H}$ to the given initial state) due to the symmetry of $\hat{H}$ in Eq. (2).

\section{EXPERIMENTAL VERIFICATION OF CAYLEY-TREE ISING PHASES}

Experiments are performed to verify the antiferrolike ground states (Phases III, IV, and V) of Cayley-tree Ising spins (see Sec. VI for technical details). Quantum annealing proceeds with three stages of time evolution, along a vertical control path from Phase I to either Phase III, IV, or V in Fig. 2. In the first stage $\left(0<t<0.1 t_{f}\right)$, Rabi frequency is slowly turned on from $\Omega_{i}=0$ to $\Omega_{0}=1.1(2 \pi) \mathrm{MHz}$, while detuning is maintained at $\Delta=-2 \Omega_{0}$ for the paramagnetic down-spin ordering $\left(\left|h_{z}^{C, V}\right| \gg J\right)$ of Phase I. In the second stage $(0.1<$ $t / t_{f}<0.9$ ), the detuning is swept from $-2 \Omega_{0}$ to $2 \Omega_{0}$, while the Rabi frequency is maintained at $\Omega_{0}$. In the final stage $\left(0.9<t / t_{f}<1\right)$, the detuning is maintained at $\Delta_{f}=2 \Omega_{0}$ (for antiferromagnetic ordering $0<\Delta_{f}<3 U$ ) and the Rabi frequency is slowly turned off from $\Omega_{0}$ to $\Omega_{f}=0$. The total operation time $t_{f}=(2 \pi) 3.2 / \Omega_{0}=2.9 \mu \mathrm{s}$ is experimentally chosen long enough to maximize the ground-state probability of $G_{10}$ within the coherence time of $10 \mu \mathrm{s}$ [43] and used for all experiments including $G_{14}$ and $G_{22}$. With the given control path, nonadiabatic leakages occur during the evolution; however, numerical simulations find that resulting excited-state probabilities are significantly smaller than the ground states for all three graphs. After the time evolution, a resulting spin configuration is detected with the fluorescence of ground-state atoms during $40 \mathrm{~ms}$ cyclic transitions to $\left|5 P_{3 / 2}, F^{\prime}=3\right\rangle$, and the procedure is repeated until the probability distribution of all spin configurations is obtained. The final Hamiltonians $\hat{H}_{G}\left(U, \Delta_{f}=2 \Omega_{0}\right)$ are chosen at various phases in Fig. 2: (1) and (2) for Phase III of $G_{10}$ and $G_{22}$, respectively; (3) for Phase IV of $G_{14}$; and (4) and (5) for Phase V of $G_{14}$.

Measured probability distributions are shown in Figs. 3(a)$3(\mathrm{c})$, which plot the probability distributions, respectively measured at (1), (2), and (4), for all spin configurations of the Cayley-tree Ising spins. Spin configurations are represented in the bare-atom basis with binary enumeration of $|\downarrow\rangle=|0\rangle$ and $|\uparrow\rangle=|1\rangle$ [45]. After the quantum annealing, the wave function of the atoms is expectedly driven near the ground state of $\hat{H}_{G}\left(U, \Delta_{f}\right)$, so the corresponding spin configuration is measured with a high probability. In Fig. 3(a), Phase III of $G_{10}$ is probed at (1). The observed max-population state is $|\uparrow ; \downarrow \downarrow \downarrow ; \uparrow \uparrow \uparrow \uparrow \uparrow \uparrow \uparrow\rangle=$ $\left|2^{9}+2^{5}+2^{4}+2^{3}+2^{2}+2^{1}+2^{0}\right\rangle=|575\rangle$, which is the antiferromagnetic phase of shell-by-shell alternating spins, agreeing with the expected ground state of $\hat{H}_{G_{10}}$ in Phase III. Likewise, in Figs. 3(b), Phase III of $G_{22}$ is probed at (2), and the observed $\left|\downarrow ; \uparrow^{3} ; \downarrow^{6} ; \uparrow^{12}\right\rangle=|1839103\rangle$ agrees with the ground state of $\hat{H}_{G_{22}}$ in Phase III. In Fig. 3(c), which shows the measurement of $G_{14}$ at (4) in Phase $\mathrm{V}$, high populations are observed for $\left|\downarrow \uparrow ; \downarrow^{4} ; \uparrow^{8}\right\rangle=|4351\rangle$ and $\left|\uparrow \downarrow ; \downarrow^{4} ; \uparrow^{8}\right\rangle=$ 


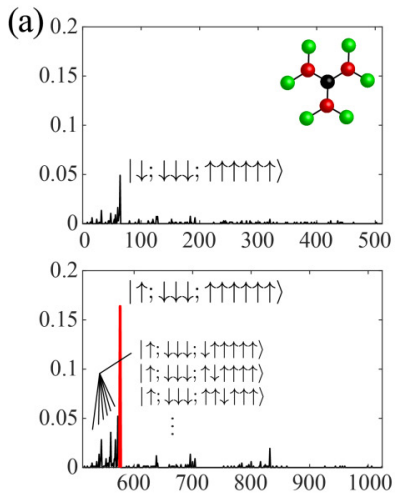

(d)

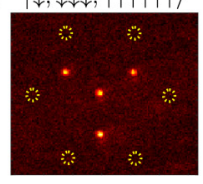

$+|\uparrow: \downarrow \downarrow \downarrow ;: \uparrow \uparrow \uparrow \uparrow \uparrow\rangle$

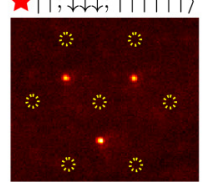

(b) 0.0

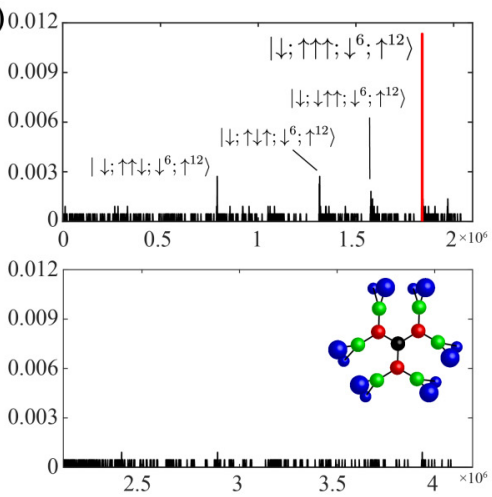

(e)

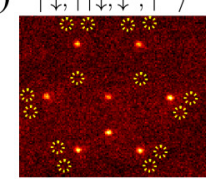

$\left|\downarrow ; \downarrow \uparrow \uparrow ; \downarrow^{6} ; \uparrow^{12}\right\rangle$

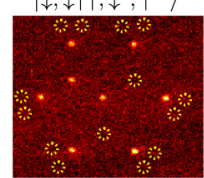

|ㅣ; $\left|\uparrow \uparrow \uparrow ; \downarrow^{6} ; \uparrow^{12}\right\rangle$

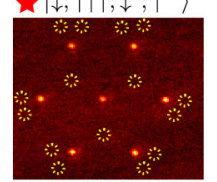

(c)
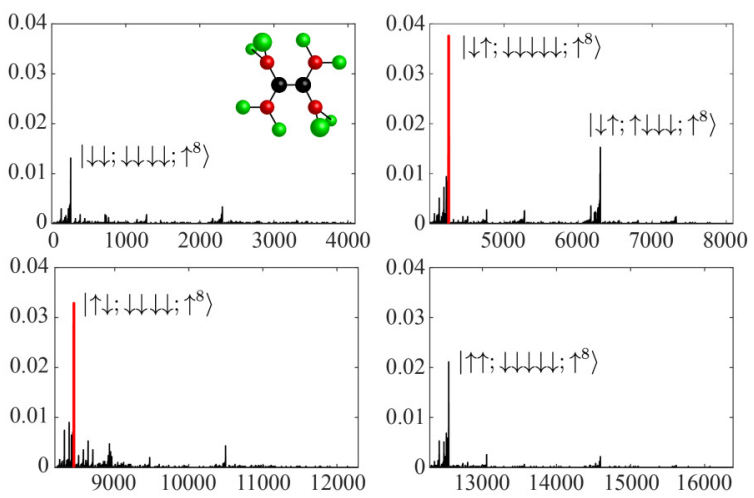

(f)
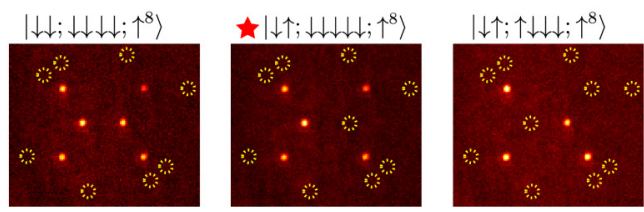
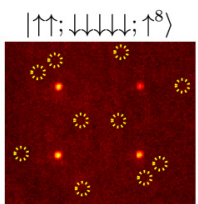

FIG. 3. Probability distribution of all spin configurations in enumerated bare-spin basis: (a) $G_{10}$ Cayley-tree atoms measured at (1) in Phase III and accumulated over 672 events. (b) $G_{22}$ at (2) in Phase III (2208 events). (c) $G_{14}$ at (4) in Phase V (5113 events). Max populations are ground states (highlighted in red), and smaller peaks are identified as near-ground excited states in numerical simulations. (d)-(f) Fluorescence images of ground-state $(|\downarrow\rangle)$ atoms in characteristic spin configurations.

$|8447\rangle$, agreeing with the ground superposition state (of center spins in $(|\uparrow \downarrow\rangle+|\downarrow \uparrow\rangle) / \sqrt{2})$ in Phase $\mathrm{V}$ of $\hat{H}_{G_{14}}$. Atom images (the fluorescence of ground-state atoms) of characteristic spin configurations are shown in Figs. 3(d)-3(f), including the max-population states (of star marks).

Phase IV of $G_{14}$ is also probed across the IV-V phase boundary, with three different Cayley trees of respective edge lengths $d / r_{b}=0.76,0.86$, and 0.92 , which correspond to (5), (4), and (3) in the phase diagram. In Fig. 4, the probabilities of three high-population states, of respective center-spin configurations $|\uparrow \uparrow\rangle,|\downarrow \downarrow\rangle$, and $(|\uparrow \downarrow\rangle+|\downarrow \uparrow\rangle) / \sqrt{2}$, are plotted. The max-populated state changes from $|\uparrow \uparrow\rangle\left(d<d_{c}=0.89 r_{b}\right.$, Phase IV) to $(|\uparrow \downarrow\rangle+|\downarrow \uparrow\rangle) / \sqrt{2}\left(d>d_{c}\right.$, Phase V), agreeing with the phase boundary given by $U\left(d_{c}\right)=\Delta_{f}$. It is noted that the higher-order, long-range couplings which are ignored in Eq. (3) play little role in the tested parameter region and that other ground states (of center-spin states $|\downarrow \uparrow\rangle,|\uparrow \downarrow\rangle$, and $(|\uparrow \downarrow\rangle-|\downarrow \uparrow\rangle) / \sqrt{2})$ are forbidden by the Hamiltonian symmetry in $\hat{H}$. Also, we observe nonadiabatic leakages to the first excited state, which changes from $(|\uparrow \downarrow\rangle+|\downarrow \uparrow\rangle) / \sqrt{2}$ to $|\downarrow \downarrow\rangle$ and then to $|\uparrow \uparrow\rangle$ from left to right, in accordance with numerical computer simulation.

\section{NÉEL'S ORDER FORMATION DYNAMICS}

Furthermore, we measure the phase formation dynamics during the quantum annealing process. Figure 5 shows the time evolution of Néel's order, defined by $O_{N} \equiv$ $-\sum_{(i, j) \in E}\left\langle\hat{\sigma}^{(i)}(t) \hat{\sigma}^{(j)}(t)\right\rangle /\|E\|$, where $\|E\|$ is the number of edges, along with the up-spin probabilities of individual atoms in $G_{10}$. With the snapshot measurements, the adiabatic order formation is clearly observed from the initial paramagnetic phase to the final antiferromagnetic phase. The oscillatory behavior is attributed to the finite size effect. In comparison, a numerical calculation (solid lines) is performed with Lindblad master equations, considering state-preparation-and-detection $($ SPAM $)$ errors $\quad(P(|\downarrow\rangle|| \uparrow\rangle)=0.18, \quad P(|\uparrow\rangle|| \downarrow\rangle)=0.02)$,

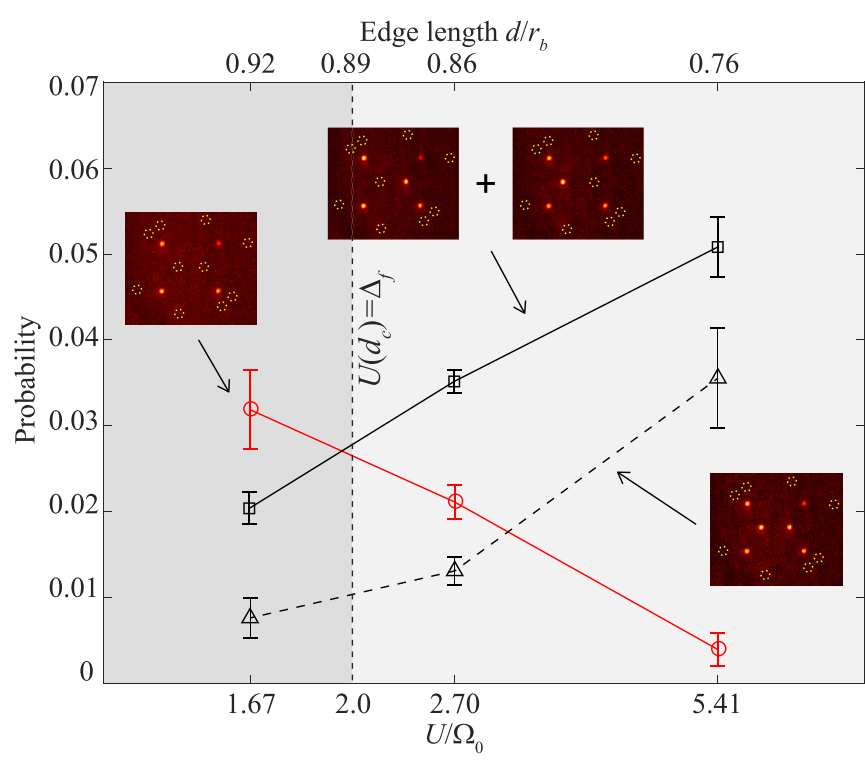

FIG. 4. Probabilities of the low-energy states of Cayley-tree Hamiltonian $\hat{H}_{G_{14}}\left(U(d), \Delta_{f}=2 \Omega_{0}\right)$ measured for three different edge lengths $d / r_{b}=0.92,0.86$, and 0.76 , which correspond to (3), (4), and (5), respectively, in the $G_{14}$ phase diagram in Fig. 2(b). 


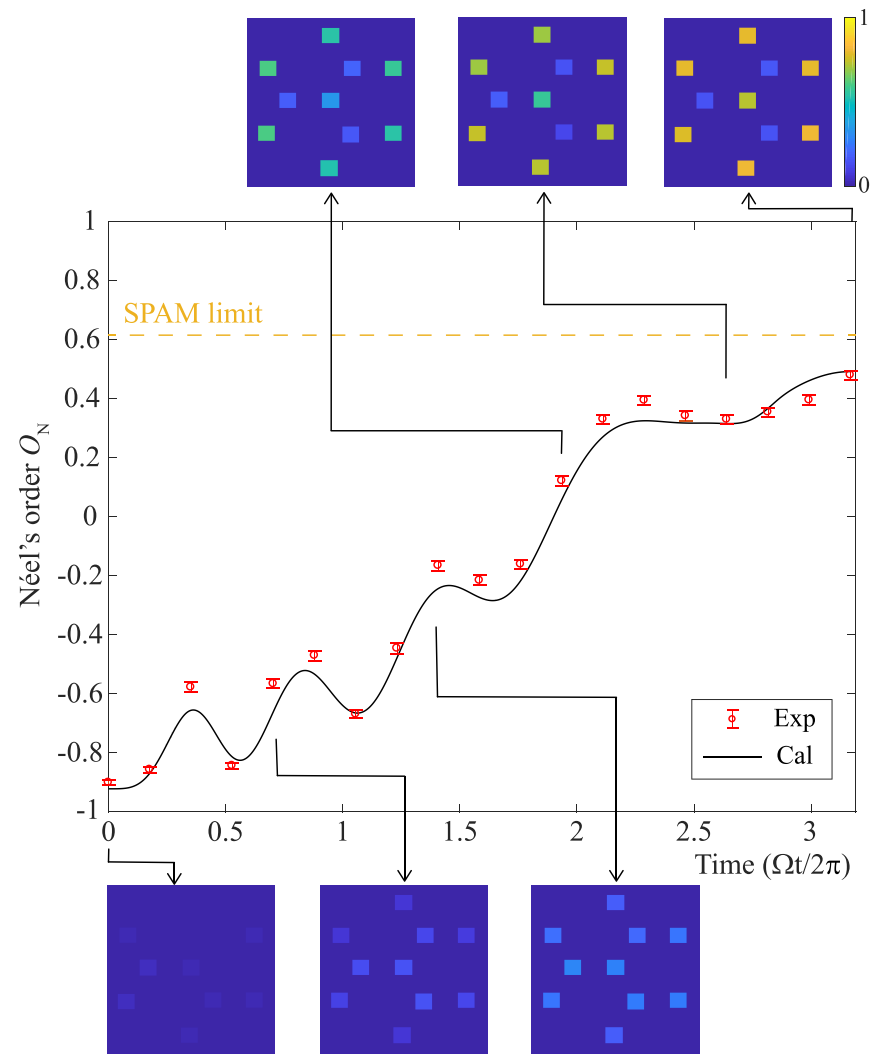

FIG. 5. Quantum annealing dynamics of the Néel's order in the $G_{10}$ Cayley tree are probed as a function of the evolution time and compared with numerical calculations. At chosen times, the Rydberg-state probabilities $\langle\hat{n}\rangle_{j}$ of all atoms $(j=1, \ldots, 10)$ are shown, being plotted at their respective atom sites.

individual dephasing $\left(\sim 36 \mathrm{kHz} \ll \Omega_{0}\right)$ due to the spontaneous decay during Rydberg excitations, and collective dephasing $(\sim 3 \mathrm{kHz})$ from laser phase noise [46]. The numerical calculation in accordance with the observed maximal Néel's order of $O_{N}\left(t_{f}\right)=0.48(2)$ indicates that errors are largely due to the SPAM errors accumulated for $N=10$ atoms. The calculation also suggests that the maximal Néel's order before measurements was $O_{N}\left(t_{f}\right)=0.82$ and that the final ground-state probability was $61 \%$.

\section{EXPERIMENTAL DETAILS}

The above experiments were performed with a Rydbergatom quantum simulator previously reported elsewhere $[43,45-47]$. In the quantum simulator, rubidium $\left({ }^{87} \mathrm{Rb}\right)$ atoms were initially prepared in the hyperfine ground state $|\downarrow\rangle=$ $\left|5 S_{1 / 2}, F=2, m_{F}=2\right\rangle$ and trapped with optical tweezers. A spatial light modulator (SLM, Meadowlark ODPDM512) was used to create the 3D array of $2 N$ optical tweezers, and an electrically focus-tunable lens (EL-16-40-TC from Optotune) verified the positions of captured atoms in all atom planes. The SLM was computer-programed with a weighted GerchbergSaxton (w-GS) algorithm $[48,49]$ so that the resulting electric field near each target site $(x, y, z)$ was created as $E(x, y, z)=$ $E_{0} \sum_{X, Y} e^{i \Phi(X, Y)} e^{-i T}$, where $X, Y$ are the SLM coordinates on the Fourier plane of the optical tweezers, $\Phi(X, Y)$ is the SLM phase pattern, and $T=2 \pi(x X+y Y) / f \lambda+$ $\pi z\left(X^{2}+Y^{2}\right) / f^{2} \lambda$ is the transfer kernel for an optical tweezer of Fresnel focal length $f$. The phase pattern $\Phi$ (for $2 N$ optical tweezers) was obtained with the w-GS condition given by $\Phi(X, Y)=\arg \left[\sum_{j=1}^{2 N} w_{j} E_{j}(x, y, z) e^{i T_{j}} /\left|E_{j}(x, y, z)\right|\right]$, where each weighting factor $w_{j}$ for $j$ th optical tweezer was optimized through adaptive iterations. The number of the wGS iterations was about five, taking about $20 \mathrm{~ms}$ to generate an array of 50 tweezers. Deterministic rearrangement of $N$ atoms to target sites, about $20 \mu \mathrm{m}$ apart from the initial atom reservoir, was performed with a consecutive 45 frames of moving traps programed with phase induction [49]. Over 90\% target-occupation probabilities were achieved in $900 \mathrm{~ms}$ reconfiguration, and all measurements were performed with defect-free arrangements.

After the atom array was prepared, the optical tweezers were temporarily turned off, and the quantum annealing proceeded. The atoms were excited to the Rydberg state $|\uparrow\rangle=$ $\left|71 S_{1 / 2}, m_{J}=\frac{1}{2}\right\rangle$ via the off-resonant intermediate state, $|i\rangle=$ $\left|5 P_{3 / 2}, F^{\prime}=3, m_{F}^{\prime}=3\right\rangle$. Two lasers $(780$ and $480 \mathrm{~nm}$ lasers for $|\downarrow\rangle \rightarrow|i\rangle$ and $|i\rangle \rightarrow|\uparrow\rangle$, respectively) were used for the two-photon transition [43]. Here, $\Omega(t)$ and $\Delta(t)$ in $H(t)$ were programed with a radiofrequency (RF) synthesizer (Moglabs XRF, $10 \mathrm{MHz}$ ) of frequency $780 \mathrm{~nm}$ and amplitude $480 \mathrm{~nm}$ modulations. The modulation ranges were $0 \leqslant \Omega(t) \leqslant \Omega_{0}$ and $-2 \Omega_{0} \leqslant \Delta(t) \leqslant 2 \Omega_{0}$, where the maximum Rabi frequency was $\Omega_{0}=\Omega_{780} \Omega_{480} /\left(2 \Delta^{\prime}\right)=1.1(2 \pi) \mathrm{MHz}$ given by $\Omega_{780}=75(2 \pi) \mathrm{MHz}, \Omega_{480}=19(2 \pi) \mathrm{MHz}$, and $\Delta^{\prime}=$ $660(2 \pi) \mathrm{MHz}$ (the intermediate detuning). The van der Waals coefficient [50] was $C_{6}=(2 \pi) 1004 \mathrm{GHz} \times \mu \mathrm{m}^{6}$ for $|71 S\rangle$ Rydberg-state atoms, and the Rydberg blockade radius was $r_{b}=\left(C_{6} / \Omega_{0}\right)^{1 / 6}=9.8 \mu \mathrm{m}$. The frequency error due to alternating current Stark shift was small, below $140 \mathrm{kHz}$, mainly caused by the $480 \mathrm{~nm}$ laser. After the quantum annealing, the optical tweezers were turned back on, and the atoms in the ground state $(|\downarrow\rangle)$ were measured whether they survived $(|\downarrow\rangle)$ or not $(|\uparrow\rangle)$. Each measurement in Fig. 3 was repeated by 672, 2208 , and 5113 times, respectively, for $G_{10}, G_{22}$, and $G_{14}$ to obtain the accumulated probability distributions.

\section{CONCLUSIONS}

We have explored the possibilities of Rydberg-atom quantum simulators toward high-dimensional qubit connection programming. With up to $N=22$ rubidium single atoms arranged in 3D space, we have programed Ising Hamiltonians on three different Cayley-tree graphs. The antiferromagnetic phase in regular Cayley trees and frustrated competing ground states in a dual-center Cayley tree are directly observed, showing good agreement with model calculations. The presented experiments were performed under the condition that uncoupled atoms are of at least $\sqrt{3}$ times larger separations than connected atoms (i.e., next-nearest-neighbor couplings are $\frac{1}{27}$ times the nearest-neighbor couplings), and this choice of atom arrangements restricts each vertex from being connected to maximally four neighboring vertices. For more complex graphs, quantum wire concepts may be necessary [51-53]. It is hoped that 3D-qubit configurations of Rydberg-atom quantum annealers shall be useful for efficient and programmable quantum optimization problems. 


\section{ACKNOWLEDGMENTS}

This paper was supported by Samsung Science and Technology Foundation (SSTF-BA1301-52), National Research
Foundation of Korea (NRF) (2017R1E1A1A01074307), and Institute for Information \& Communications Technology Promotion (IITP-2018-2018-0-01402).
[1] E. Farhi, J. Goldstone, S. Gutmann, J. Lapan, A. Lundgren, and D. Preda, A quantum adiabatic evolution algorithm applied to random instances of an NP-complete problem, Science 292, 472 (2001).

[2] A. Das and B. K. Chakrabarti, Colloquium: Quantum annealing and analog quantum computation, Rev. Mod. Phys. 80, 1061 (2008).

[3] T. Albash and D. A. Lidar, Adiabatic quantum computation, Rev. Mod. Phys. 90, 015002 (2018).

[4] P. Hauke, H. G. Katzgraber, W. Lechner, H. Nishimori, and W. D. Oliver, Perspectives of quantum annealing: methods and implementations, Rep. Prog. Phys. 83, 054401 (2020).

[5] M. W. Johnson et al., Quantum annealing with manufactured spins, Nature 473, 194 (2011).

[6] P. I. Bunyk, E. M. Hoskinson, M. W. Johnson, E. Tolkacheva, F. Altomare, A. J. Berkley, R. Harris, J. P. Hilton, T. Lanting, A. J. Przybysz, and Jed Whittaker, Architectural considerations in the design of a superconducting quantum annealing processor, IEEE Trans. Appl. Supercond. 24, 1 (2014).

[7] D. Rosenberg, D. Kim, R. Das, D. Yost, S. Gustavsson, D. Hover, P. Krantz, A. Melville, L. Racz, G. O. Samach, S. J. Weber, F. Yan, J. L. Yoder, A. J. Kerman, and W. D. Oliver, 3D integrated superconducting qubits, Npj Quantum Inf. 3, 42 (2017).

[8] M. P. Harrigan et al., Quantum approximate optimization of non-planar graph problems on a planar superconducting processor, Nat. Phys. 17, 332 (2021).

[9] S. Korenblit, D. Kafri, W. C. Campbell, R. Islam, E. E. Edwards, Z.-X. Gong, G.-D. Lin, L.-M. Duan, J. Kim, K. Kim, and C. Monroe, Quantum simulation of spin models on an arbitrary lattice with trapped ions, New J. Phys. 14, 095024 (2012).

[10] R. Islam, C. Senko, W. C. Campbell, S. Korenblit, J. Smith, A. Lee, E. E. Edwards, C.-C. J. Wang, J. K. Freericks, and C. Monroe, Emergence and frustration of magnetism with variable-range interactions in a quantum simulator, Science 340, 583 (2013).

[11] P. Richerme, C. Senko, J. Smith, A. Lee, S. Korenblit, and C. Monroe, Experimental performance of a quantum simulator: Optimizing adiabatic evolution and identifying many-body ground states, Phys. Rev. A 88, 012334 (2013).

[12] P. Hauke, L. Bonnes, M. Heyl, and W. Lechner, Probing entanglement in adiabatic quantum optimization with trapped ions, Front. Phys. 3, 21 (2015).

[13] R. Babbush, P. J. Love, and A. Aspuru-Guzik, Adiabatic quantum simulation of quantum chemistry, Sci. Rep. 4, 6603 (2014).

[14] H. Neven, V. S. Denchev, G. Rose, and W. G. Macready, Training a binary classifier with the quantum adiabatic algorithm, arXiv:0811.0416.

[15] E. G. Rieffel, D. Venturelli, B. O’Gorman, M. B. Do, E. M. Prystay, and V. N. Smelyanskiy, A case study in programming a quantum annealer for hard operational planning problems, Quant. Inf. Proc. 14, 1 (2015).
[16] K. C. Young, M. Sarovar, and R. Blume-Kohout, Error Suppression and Error Correction in Adiabatic Quantum Computation: Techniques and Challenges, Phys. Rev. X 3, 041013 (2013).

[17] S. Boixo, T. F. Rønnow, S. V. Isakov, Z. Wang, D. Wecker, D. A Lidar, J. M. Martinis, and M. Troyer, Evidence for quantum annealing with more than one hundred qubits, Nat. Phys. 10, 218 (2014).

[18] K. L. Pudenz, T. Albash, and D. A. Lidar, Error-corrected quantum annealing with hundreds of qubits, Nat. Commun. 5, 3243 (2014).

[19] S. Ebadi, T. T. Wang, H. Levine, A. Keesling, G. Semeghini, A. Omran, D. Bluvstein, R. Samajdar, H. Pichler, W. W. Ho, S. Choi, S. Sachdev, M. Greiner, V. Vuletić, and M. D. Lukin, Quantum phases of matter on a 256-atom programmable quantum simulator, arXiv:2012.12281.

[20] P. Scholl, M. Schuler, H. J. Williams, A. A. Eberharter, D. Barredo, K.-N. Schymik, V. Lienhard, L.-P. Henry, T. C. Lang, T. Lahaye, A. M. Läuchli, and A. Browaeys, Programmable quantum simulation of $2 \mathrm{D}$ antiferromagnets with hundreds of Rydberg atoms, arXiv:2012.12268.

[21] B. Altshuler, H. Krovi, and J. Roland, Anderson localization makes adiabatic quantum optimization fail, Proc. Natl. Acad. Sci. USA 107, 12446 (2010).

[22] R. D. Somma, D. Nagaj, and M. Kieferová, Quantum Speedup by Quantum Annealing, Phys. Rev. Lett. 109, 050501 (2012).

[23] T. F. Rønnow, Z. Wang, J. Job, S. Boixo, S. V. Isakov, D. Wecker, J. M. Martinis, D. A. Lidar, and M. Troyer, Defining and detecting quantum speedup, Science 345, 420 (2014).

[24] S. Muthukrishnan, T. Albash, and D. A. Lidar, Sensitivity of quantum speedup by quantum annealing to a noisy oracle, Phys. Rev. A 99, 032324 (2019).

[25] W. Lechner, P. Hauke, and P. Zoller, A quantum annealing architecture with all-to-all connectivity from local interactions, Sci. Adv. 1, e1500838 (2015).

[26] A. W. Glaetzle, R. M. van Bijnen, P. Zoller, and W. Lechner, A coherent quantum annealer with Rydberg atoms, Nat. Commun. 8, 15813 (2017).

[27] H. Pichler, S.-T. Wang, L. Zhou, S. Choi, and M. D. Lukin, Quantum optimization for maximum independent set using Rydberg Atom Arrays, arXiv:1808.10816.

[28] H. Weimer, M. Müller, I. Lesanovsky, P. Zoller, and H. P. Büchler, A Rydberg quantum simulator, Nat. Phys. 6, 382 (2010).

[29] A. Broweays and T. Lahaye, Many-body physics with individually controlled Rydberg atoms, Nat. Phys. 16, 132 (2020).

[30] W. Lee, H. Kim, and J. Ahn, Three-dimensional rearrangement of single atoms using actively controlled optical microtraps, Opt. Express 24, 9816 (2016).

[31] H. Kim, W. Lee, H.-g. Lee, H. Jo, Y. Song, and J. Ahn, In situ single-atom array synthesis by dynamic holographic optical tweezers, Nat. Commun. 7, 13317 (2016). 
[32] D. Barredo, S. de Léséleuc, V. Lienhard, T. Lahaye, and A. Browaeys, An atom-by-atom assembler of defect-free arbitrary 2D atomic arrays, Science 354, 1021 (2016).

[33] M. Endres, H. Bernien, A. Keesling, H. Levine, E. R. Anschuetz, A. Krajenbrink, and M. D. Lukin, Atom-by-atom assembly of defect-free one-dimensional cold atom arrays, Science 354, 1024 (2016).

[34] D. Barredo, V. Lienhard, S. de Léséleuc, T. Lahaye, and A. Browaeys, Synthetic three-dimensional atomic structures assembled atom by atom, Nature 561, 79 (2018).

[35] T. Pohl, E. Demler, and M. D. Lukin, Dynamical Crystallization in the Dipole Blockade of Ultracold Atoms, Phys. Rev. Lett. 104, 043002 (2010).

[36] P. Schauss, J. Zeiher, T. Fukuhara, S. Hild, M. Cheneau, T. Macri, T. Pohl, I. Bloch, and C. Gross, Crystallization in Ising quantum magnets, Science 347, 1456 (2015).

[37] H. Bernien, S. Schwartz, A. Keesling, H. Levine, A. Omran, H. Pichler, S. Choi, A. S. Zibrov, M. Endres, M. Greiner, V. Vuletić, and M. D. Lukin, Probing many-body dynamics on a 51-atom quantum simulator, Nature 551, 579 (2017).

[38] V. Lienhard, S. de Léséleuc, D. Barredo, T. Lahaye, A. Browaeys, M. Schuler, L.-P. Henry, and A. M. Läuchli, Observing the Space- and Time-Dependent Growth of Correlations in Dynamically Tuned Synthetic Ising Models with Antiferromagnetic Interactions, Phys. Rev. X 8, 021070 (2018).

[39] I. I. Beterov, D. B. Tretyakov, V. M. Entin, E. A. Yakshina, I. I. Ryabtsev, M. Saffman, and S. Bergamini, Application of adiabatic passage in Rydberg atomic ensembles for quantum information processing, J. Phys. B: At. Mol. Opt. Phys. 53, 182001 (2020).

[40] H. A. Bethe, Statistical theory of superlattices, Proc. Roy. Soc. A 150, 552 (1935).

[41] M. Ostilli, Cayley trees and Bethe lattices: A concise analysis for mathematicians and physicists, Physica A 391, 3417 (2011).
[42] R. J. Baxter, Exactly Solvable Models in Statistical Mechanics (Academic Press, London, 1982).

[43] M. Kim, Y. Song, J. Kim, and J. Ahn, Quantum-Ising Hamiltonian programming in trio, quartet, and sextet qubit systems, PRX Quantum 1, 020323 (2020).

[44] A. Lucas, Ising formulations of many NP problems, Front. Phys. 2, 5 (2014).

[45] H. Kim, Y. J. Park, K. Kim, H.-S. Sim, and J. Ahn, Detailed Balance of Thermalization Dynamics in Rydberg Atom Quantum Simulators, Phys. Rev. Lett. 120, 180502 (2018).

[46] W. Lee, M. Kim, H. Jo, Y. Song, and J. Ahn, Coherent and dissipative dynamics of entangled few-body systems of Rydberg atoms, Phys. Rev. A 99, 043404 (2019).

[47] H. Jo, Y. Song, M. Kim, and J. Ahn, Rydberg Atom Entanglements in the Weak Coupling Regime, Phys. Rev. Lett. 124, 033603 (2020).

[48] R. Di Leonardo, F. Ianni, and G. Ruocco, Computer generation of optimal holograms for optical trap arrays, Opt. Express 15, 1913 (2007).

[49] H. Kim, M. Kim, W. Lee, and J. Ahn, Gerchberg-Saxton algorithm for tweezer-trap atom arrangements, Opt. Express 27, 2184 (2019).

[50] S. Weber, C. Tresp, H. Menke, A. Urvoy, O. Firstenberg, H. P. Büchler, and S. Hofferberth, Tutorial: Calculation of Rydberg interaction potentials, J. Phys. B: At. Mol. Opt. Phys. 50, 133001 (2017).

[51] S. Diehl, E. Rico, M. A. Baranov, and P. Zoller, Topology by dissipation in atomic quantum wires, Nature Physics 7, 971 (2011).

[52] M. F. Serret, B. Marchand, and T. Ayral, Solving optimization problems with Rydberg analog quantum computers: Realistic requirements for quantum advantage using noisy simulation and classical benchmarks, arXiv:2006.11190.

[53] X. Qiu, P. Zoller, and X. Li, Programmable quantum annealing architectures with Ising quantum wires, PRX Quantum 1, 020311 (2020). 